\title{
Kirchliche Sendungen im Fernsehen am Beispiel des ZDF: \\ Redaktion Kirche und Leben (katholisch)
}

von Michael Albus

Der Programmauftrag, der mit dem Titel „Kirche und Leben” gegeben ist, erfordert es, im Programm die Spannung zu verdeutlichen, die in den beiden Bereichen Kirche und Leben schon von sich aus gegeben ist. Die Spannung ist hier im doppelten Sinne zu interp retieren: einmal als Spannung, die Voraussetzung für einen echten Fortschritt und eine wirkliche Hilfe ist, zum anderen Spannung, die auch Belastung und Konflikt andeutet.

\section{Grundsätzliche Bemerkungen zum Programmauftrag der Redaktion Kirche und Leben} (kath.)

Diese doppelte Spannung verbietet, daß sich das Programm der Redaktion etwa nur mit gesellschaftspolitischen Fragen befassen darf. Es kommt vor allem darauf an, daß die sozialpolitischen und sozial-ethischen Themen, die in der Praxis sicher im Vordergrund stehen, vom Christlichen her gesehen und verstehbar gemacht werden müssen. Daraus ergeben sich folgende vier grundsätzliche Konsequenzen für das Programm unserer Redaktion:

1. Lebenshilfe, Sinndeutung, „Seelsorge” ohne den direkten pastoralen Effekt, aber nicht ohne pastorales Element. Es geht darum, Verstehenshorizonte für die heutigen Fragen aus der Sicht des Christentums und der Kirche zu eröffnen, Fragen und Lösungsmöglichkeiten aufzuzeigen, die sicher in vielem - aus der christlichen Botschaft heraus - quer zu gängigen Fragen und Lösungsmöglichkeiten stehen, die Antworten über den Tag hinaus zeigen.

2. Kritische Möglichkeit geben zu einer solidarischen Identifikation mit der Kirche. Die Kirche gehört zum Glauben dazu. Kritisch sein heißt hier: die Impulse des Evangeliums, auf das die Kirche gründet, müssen im Handeln der Institution erkennbar und überprüfbar sein. Eigentlich müßte das Programm als ganzes gesehen in dieser Hinsicht Mut machen zum konkreten Mehrtun und Einsatz im kirchlichen wie im gesellschaftlichen Bereich.

3. Korrektur einseitiger Informationen über den Glauben im Sinne einer Auseinandersetzung mit anderen Sinn- und Lebensentwürfen als den christlichen.

4. Sinnvermittlung durch das Vermitteln von „Transzendenz-Erfahrung” als Grundlage zum Aufnehmen der religiösen Lebensfrage. Hier sind im Augenblick starke Tendenzen vor allem bei Jugendlichen festzustellen, die in einer ganz neuen Art auf diese Fragen zugehen. Letzlich bedeutet dies Hilfe zur Sprengung von Ideologien oder „Heilslehren”, die den Menschen in seinem Ganzsein bedrohen.

2. Grundsätzliche Probleme, die sich bei der Durchführung dieses Auftrags ergeben.

Grundsätzliche Probleme, die sich bei der Durchführung dieses Auftrags ergeben, sehe ich in folgendem:

Dr. Michael Albus ist verantwortlicher Redakteur der Redaktion Kirche und Leben (katholisch) beim Zweiten Deutschen Fernsehen in Mainz. 
- Esist sowohl im gesellschaftlichen Bereich als auch in einer Anstalt öffentlichen Rechts wie dem ZDF selbst heute nicht mehr ganz einfach, die Notwendigkeit der oben angedeuteten Fragestellungen einsichtig zu machen. Dies liegt zum einen an dem Säkularisierungs-Prozeß der letzten 20 Jahre, der neben manchen positiven Aspekten auch eine eindeutige Tendenz zur Abwehr kirchlicher Fragestellungen aufweist. Zum anderen liegt es aber auch an einer fühlbaren Schwäche der Kirchen selbst. Ihre innere Autorität hat nachgelassen, und die Aufbrüche, die neue Wege andeuten, sind noch keine Durchbrüche. In einer solchen Lage fälltein entscheidender Anteilan Durchsetzungsarbeit in eineröffentlich rechtlichen Anstalt den Redakteuren selbst zu. Themen stehen und fallen mit der Kraft bzw. Kraftlosigkeit ihrer Argumente im internen Diskussionsprozeß. Im Blick auf die Kirche als Institution heißt das sehr oft: Wir müssen - nach Heinrich Böll - unser Brot „selber backen und das Wort uns selbst bereiten" (Brief an einen jungen Katholiken, 1958) - leider.

- Ein Theorie-Defizit des Programms in den vergangenen Jahren wirkt sich besonders auch in unserer Redaktion aus. Man hat allzu leichtfertig, was die Programminhalte angeht, nach meiner Meinung jahrelang von der Hand in den Mund gelebt. Man hat kaum daran gedacht, eine fundierte Systematik der Erarbeitung der Themen und Inhalte des Programms einzuführen. Ich glaube jedoch, daß ein gutes Programm auf die Dauer auf diesen wenig sichtbaren, aber entscheidend wichtigen Arbeitskomplex nicht verzichten kann. Ich bin der Überzeugung, daß eine konsequente Arbeit im Vorfeld des Programms sich beim Zuschauer erkennbar auswirkt.

- Die vom Programmschema her für unsere meist sehr komplexen und vielschichtigen Themen vorgegebenen Sendezeiten sind oft nicht ausreichend. Ich bin kein Anhänger der weitverbreiteten Meinung, daß eine Verlängerung der Sendezeit immer den Erfolg bringt, der durch eine mangelnde inhaltliche Arbeit ausgeblieben ist oder ausbleibt. Aber, um bei einem Beispiel zu bleiben: Eine Sendezeit von real 28 Minuten zur Darstellung etwa des Problems der Schuld oder der Angst des Menschen von heute ist faktisch nicht ausreichend. Die Folge davon ist, daß wir oft dazu gezwungen werden, wichtige Aspekte aus Zeitgründen auszuklammern. Zahlreiche Zuschauerreaktionen weisen auf diesen Mangel hin. Sicher müssen alle Anstrengungen unternommen werden, die bestehenden Sendezeiten inhaltlich und formal besser auszufüllen. Aber danach stellt sich dann doch wieder die Frage, ob für manche Themen, wie sie gera de beiuns anfallen, nicht doch etwas größere Zeiteinheiten größere Chancen bringen, größere Möglichkeiten zur ruhigen Ausbreitung eines differenzierten Stoffs eröffnen würden.

Für unsere Redaktion erweist es sich als negativ, daß in der Anstalt großer Wert auf die Einschaltquoten und damit wohl auf eine vorwiegend quantitative Bewertung von Fragen gelegt wird, deren Bedeutung so nicht meßbar ist. Auch hier gilt: daß solchen quantitativen Beurteilungskriterien in einer Anstalt nur durch die Flucht in die Qualität der Sendungen begegnet werden kann.

\section{Programmschwerpunkte für den Zeitraum 1977 - 1980.}

Aufgrund einer von verschiedenen Richtungen herkommenden fortlaufenden Analyse der kirchlichen und gesellschaftlichen Entwicklung ergeben sich für den Zeitraum 1977-'80 in unserer Redaktion folgende grundsätzliche Programmschwerpunkte:

1. Die Diskussion um die Menschenrechte (international) und die Grundwerte (vorwiegend national). Begriffe wie Freiheit, Gerechtigkeit, Solidarität, Verantwortung, Bindung müssen an einzelnen Beispielen verdeutlicht werden. Die Leitlinie heißt hier: 
nicht über die Menschenrechte und Grundwerte diskutieren, sondern beispielhafte und im Sinne einer Hilfestellung für den Zuschauer fortführende einzelthematische Darstellungen in's Programm bringen.

2. Der ethische, soziale und religiöse Beitrag der Kirchen zu gesellschaftspolitischen Grundfragen in unserem Land. Hier kommen auf Grund der gegenwärtigen Entwicklung vor allem die Bereiche Ehe, Familie, Medien und Erziehung in Frage.

3. Die Auseinandersetzung zwischen grundlegenden und auch grundlegend verschiedenen Weltanschauungen wie Christentum und Marxismus - Sozialismus, aber auch etwa Buddhismus - Islam. Dieser Programmschwerpunkt wird besonders im Bereich der Dokumentation und des Tagebuchs aus der Katholischen Kirche zu berücksichtigen sein.

4. Eine fortlaufende Darstellung der kirchlichen Entwicklung auf nationaler und Weltebene. Dabei steht die Frage im Vordergrund, wie sich die Kirche auf die oben angedeuteten Probleme einstellt. Ob sie heute eine lebbare Alternative zu anderen „Heilsentwürfen" bieten kann, ob es Aufbrüche in ihr dahin gibt, welchen Beitrag sie also zur Sinnfrage leistet?

5. Entwicklung und Mission. Der Beitrag der Kirche zu den Menschenrechten in der Dritten Welt. Dieser Schwerpunkt wird vor allem wichtig im Blick auf die Tatsache, daß die bisherigen Formen auch kirchlicher Entwicklungshilfe den Wünschen und Notwendigkeiten der Menschen in den dortigen Ländern nicht mehr voll gerecht werden. $\mathrm{Da}$ es heute auch hier vor allem um die Entwicklung von Inhalten geht. Vor welchen Problemen steht nun - eine mögliche Frage - die kirchliche Mission angesichts des Umbruchs politischer Systeme in den „alten” Missionsländern, aber auch angesichts eines neuen religiösen Aufbruchs bei uns, der außerhalb der Kirchen und oft an ihnen vorbei geschieht?

\section{Sendeformen}

Im Augenblick sind jährlich folgende Sendungen in der Verantwortung der Redaktion:

1. 6 x Spielfilme á 45 Minuten - als Spielfilmreihe.

Die Sendezeit ist augenblicklich noch Sonntagabend 19.30 Uhr. Eine Rückkehr zu dem ursprünglichen Sendetermin von Mittwochabend ist vorgesehen.

2. $4 \mathrm{x}$ KONTAKTE - Magazin für Lebensfragen à 45 Minuten.

Sendezeit: montags $20.15 \mathrm{Uhr}$.

3. $13 \times$ Dokumentationen à 30 Minuten.

Sendezeit: mittwochs 22.05.

4. $26 \times$ Tagebuch aus der katholischen Kirche.

Sendezeit: Sonntag 18.00 - 18.15 Uhr.

5. $12 \times$ Gottesdienste/live à 45 Minuten.

Sendezeit: Sonntag $9.15-10.00 \mathrm{Uhr}$.

6. 6 x Feiertags-Sendungen à 15 Minuten an den entsprechenden Feiertagen.

Sendezeit: 19.15 Uhr. 
Die Sendezeit-Statistik des ZDF im Zeitraum 1. Januar bis 31 Dezember 1978 weist für insgesamt 70 von der katholischen Redaktion verantwortete Sendungen im Jahr 2.274 Sendeminuten aus.

Nimmt man noch die Sendungen in der Verantwortung der evangelischen Redaktion hinzu, dann haben wir für das Jahr 1976 insgesamt 3.775 Sendeminuten. Das sind rund 10\% der Sendeminuten der Hauptabteilung Kultur, zu der die beiden kirchlichen Redaktionen im ZDF gehören.

Es ist bei der Beantwortung der Frage nach dem Stellenwert kirchlicher Sendungen im Gesamtprogramm des ZDF entscheidend, von welchen Vorstellungen man ausgeht. Sicher wird manchen der quantitative Stellenwert kirchlicher Sendungen im Gesamtprogramm zu gering sein, sie wünschten sich mehr Sendezeit. Ich halte außer in einigen wohlbegründeten Fällen nichts davon, wenn man sich von quantitativen Erweiterungen des Programms etwas verspricht. Es sollte vielmehr das Bestreben der Redaktion im Vordergrund stehen, qualitative Verbesserungen innerhalb der vorgegebenen Sendezeit zu erreichen, und es sollte das Bestreben der Kirche sein, auf dem Wege der Ausführung und Erfüllung des Staatsvertrages darauf zu achten, beizeiten inhaltliche Begründungen - nicht nur Berufung auf juristisch fixierte Positionen - parat zu haben, die das Schrumpfen der Sendezeiten und die Verschlechterung der Sendetermine verhindern. Beides, die Arbeit der Redaktion und das Bemühen der Kirche, hängen in diesem Fall eng miteinander zusammen. Insgesamt wird man sagen dürfen, daß der Stellenwert kirchlicher Sendungen im Gesamtprogramm des ZDF nach wie vor nicht schlecht ist. Aber das kann sich ändern, und darauf sollte man vorbereitet sein - in jeder Beziehung.

An dieser Stelle noch ein paar Sätze zum Verhältnis Kirche und Fernsehen; also dazu, wie man auf verantwortlicher kirchlicher Seite zu unserer Arbeit steht - nach meiner Einschätzung.

Es gibt da eine Menge guter und positiver Zusammenarbeit, etwa mit dem kírchlichen Beauftragten beim ZDF, und von Seiten der Anstalt dürfte in den wesentlichsten Positionen ebenfalls Positives gelten.

Wo ich nach wie vor Wünsche habe: die Einstellung einer Reihe von kirchlichen Amtsträgern und Stellen zu unserer journalistischen Arbeit, zu unseren Sendungen.

Da ist mir noch zuviel pauschale Beurteilung oder Verurteilung, zuwenig Sachkunde. Zuviel Kritik an Formalem, zuwenig Argumentation zum Inhaltlichen. Zuviel Sporadisches, zuwenig Kontinuierliches; das heißt: Schweigen bei Zustimmung, Briefe, halböffentliche Äußerungen bei Ablehnung. Und auch zuwenig Beteiligung am täglichen Informationsfluß. Beispiel etwa das Sekretariat der Deutschen Bischofskonferenz. Man muß sich seine Informationen überall herholen, weil man sie von dort nur selten bekommt. Zuviel Absichtserklärung, zuwenig Verwirklichung. Ich bin der Meinung, daß, wenn man „communio et progressio" wirklich emst nimmt, die eben genannte Wunschliste schlagartig kürzer wird.

Wir bemühen uns jedenfalls von Seiten der Redaktion in Zusammenarbeit mit dem kirchtichen Beauftragten beim ZDF nach Kräften darum, zu unseren Zuschauern Kontakt zu halten und neue Kontakte zu bekommen. So kann ich etwa darauf hinweisen, daß die für das nächste Jahr geplanten vier Themen der Reihe KONTAKTE-Magazin für Lebensfragen zu einem großen Teil das Ergebnis einer intensiven Auswertung der in 
die Tausende gehenden Zuschauerbriefe auf die Sendungen des vergangenen Jahres sein werden. Aber darüberhinaus gibt es noch eine Reihe anderer, noch vielleicht zu entdeckender oder auszubauender Kontakt-Möglichkeiten mit unseren Zuschauern.

\section{SUMMARY}

In his capacity as Editor of Catholic Programmes of the "Church and Life Department" (Religious section of the German TV Second channel ZDF), the author speaks from experience. Sociopolitical and socio-ethical themes dominate the programming of this section, but they have to be seen and interpreted in a Christian perspective. This has practical, concrete consequences. The problems in trying to follow this principle are obvious, so he gives critical comments on both the quantity and the quality of programmes and his views measu red against typical examples taken from between 1977 and 1980. Responsible people in the Church are asked not to make generalisations or condemnations without sufficient professional insight ... If they were to take 'Communio et Progressio' more seriously, some of the lack of understanding between the Church and TV would disappear.

\section{RÉSUMÉ}

L'auteur prend partie, de façon très décidée, sur les émissions religieuses à la télévision: à partir de sa propre expérience en tant que rédacteur responsable de "l'Eglise et la vie», sur la deuxième chaine, du côté catholique. A partir de la mission du programme, il délimite que les thèmes sociaux-politiques et socio-éthiques qui se trouvent pratiquement au premier plan doivent être vus du point de vue chrétien et rendus compréhensibles à tous. A partir de cela, il développe quelques conséquences pratiques. Il montre également les problèmes qui résultent de l'exécution de cette mission. A l'aide des problèmes centraux du programme pour la période 1977 à 1980 et à l'aide des formes d'émission, il développe ses remarques critiques quant à la quantité et à la qualité des émissions. Il émet ses souhaits par une série des représentants officiels de l'Eglise: trop de critique en bloc ou condamnation, trop peu de compétence etc. .. Il pense que la liste des souhaits diminuerait dès qu'on prendrait «Communio et Progressio» vraiment au sérieux.

\section{RESUMEN}

El autor adopta una postura decididamente crítica ante las emisiones religiosas de la Televisión, partiendo de su propia experiencia como redactor responsable de "Iglesia y Vida", en la Segunda Cadena de Televisión. Con respecto a la programmación, señala que, los temas socio-políticos y ético-sociales, que gozan en la práctica de preeminencia, deben ser tratados desde una perspectiva cristiana. De ello saca consecuencias prácticas. Apunta también a los problemas que surgen de la realización. A tenor de los puntos básicos para el lapso 1977 a 1980, y teniendo como punto de referencia las formas de emisión, desarrolla sus anotaciones críticas sobre la cantidad y calidad de las emisiones. Expresa deseos remitidos a una serie de miembros de la jerarquía eclesiástica, quejándose de juicios o condenas globales, de poco conocimiento de la materia. La lista de deseos se reduciría, opina, en cuanto se tomara en serio la "Communio et Progressio". 